\title{
Numerical Analysis of Strain-Induced Surface Phenomena in Aluminum Alloys
}

\author{
Varvara Romanova ${ }^{1,2, a)}$, Ruslan Balokhonov ${ }^{1,2, b)}$, \\ Olga Zinovieva, ${ }^{1,3}$, and Ekaterina Batukhtina ${ }^{3}$ \\ ${ }^{1}$ Institute of Strength Physics and Materials Science SB RAS, Tomsk, 634055, Russia \\ ${ }^{2}$ National Research Tomsk Polytechnic University, Tomsk, 634050, Russia \\ ${ }^{3}$ National Research Tomsk State University, Tomsk, 634050, Russia

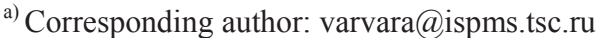 \\ b) rusy@ispms.tsc.ru
}

\begin{abstract}
Mesoscale surface deformation in polycrystalline aluminum alloys subjected to uniaxial tension is numerically investigated. Three-dimensional polycrystalline models with equiaxial and extended grains peculiar to rolling are constructed by a step-by-step packing method. Calculation results have shown that the grain structure is responsible for the mesoscale surface roughening under uniaxial tension. The roughness pattern is affected by the microstructure and loading conditions. In a specimen with equiaxial grains and in a textured material loaded across the rolling direction surface relief is very pronounced in comparison with the extended grain structure loaded along rolling direction.
\end{abstract}

Keywords: microstructure-based simulation, three-dimensional models, mesoscale surface roughening, uniaxial tension

\section{INTRODUCTION}

Aluminum alloys due to their high strength and plasticity characteristics in combination with light weight are widely used in car and aircraft industry. In some cases, however, their application is limited to the morphological changes occurring on the free surface under deformation. This phenomenon known as strain-induced surface roughening is unwanted effect resulting in cosmetic defects and in impairment of mechanical properties. Experimental and numerical investigations of roughening mechanisms are necessary to develop effective methods to control roughening.

Extensive experimental and numerical data on the effects of grain size, texture and loading conditions on the roughening in aluminum alloys are published at present (e.g., [1, 2]). Roughening is treated as a multiscale phenomenon evolving simultaneously at micro-, meso- and macroscales [3]. Our study is devoted to the mesoscale effects where the grain structure plays a key role. A characteristic feature of the mesoscale roughening is that it is not individual grains but grain ensembles are involved in cooperative displacements to form ridges and valleys on the free surface. Although microscopic mechanisms of local plastic deformation are attributed to dislocation motion, the mesoscale roughening is apparently controlled by a larger scale inhomogeneity and cannot be described in terms of dislocation theory alone. In polycrystalline metals the mesoscale deformation is attributed to the grain structure. It is, therefore, a motivation to analyze surface roughening using microstructure-based models.

In this paper the effects of the grain shape, texture and loading conditions on the surface roughness characteristics are numerically investigated using two polycrystalline models with extended and equiaxial grains. The mechanical aspects of the surface roughness evolution and its correlation with the internal stress-strain state are analyzed and discussed.

International Conference on Physical Mesomechanics of Multilevel Systems 2014

AIP Conf. Proc. 1623, 527-530 (2014); doi: 10.1063/1.4898998

(C) 2014 AIP Publishing LLC 978-0-7354-1260-6/\$30.00 


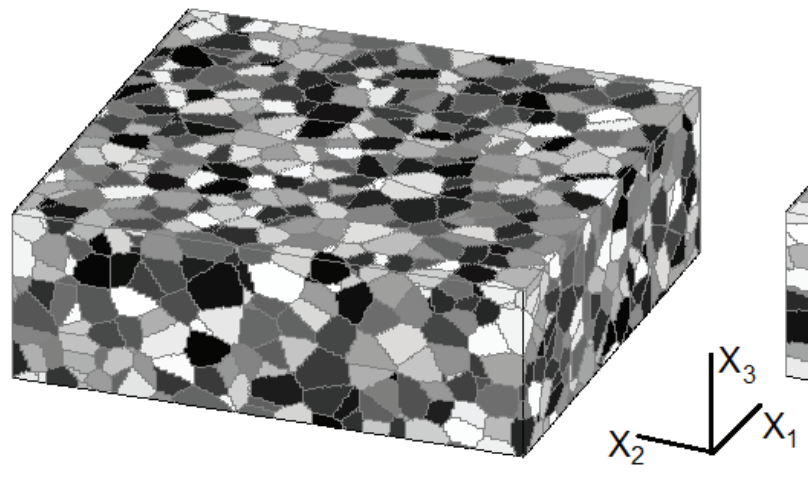

(a)

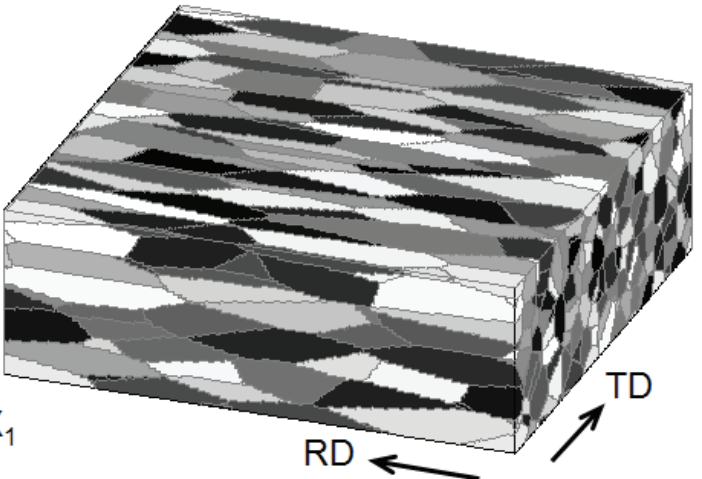

(b)

FIGURE 1. Polycrystalline models with equiaxial (a) and extended grains (b)

\section{MICROSTRUCTURE-BASED SIMULATION}

The procedure of a 3D numerical analysis using the finite-difference method is detailed elsewhere (see, e.g., [4]). We consider only briefly major simulation stages.

Simulation procedure starts with the development of a microstructure-based constitutive model which is incorporated into a general system of equations of continuum mechanics, including laws of conservation of mass, momentum, and energy. The system of equations supplemented with initial and boundary conditions is solved numerically by the finite-difference method [4].

In this work, the three-dimensional mechanical problem is solved numerically by the finite-difference method using a regular cubic mesh. Polycrystalline models were constructed using a step-by-step packing method [5] to design three-dimensional microstructures of different types. Generally, the step-by-step packing design to reproduce a polycrystalline structure includes the following steps. A 3D volume is discretized by a fine regular cubic mesh and three-dimensional coordinates are defined for the mesh nodes. A number of computational cells assigned randomly or by a given law are treated as grain nuclei. At each step in the design procedure, the volumes surrounding the nuclei are incremented by preset values in accordance with given growth laws. The growing grains are complemented by the mesh elements falling within the incremented zones. The procedure is repeated until growing structure to cover the entire volume.

In this work, the step-by-step packing procedure was applied in designing periodical polycrystalline microstructures with equiaxial and extended grains inherent in cold rolling. In the former case, all grains were grown at the same growth rate in accordance with a spherical law. To design a microstructure with extended grains oriented along the rolling direction (RD) an ellipsoidal law of grain growth was used with an aspect ratio of ellipsoid semiaxes being $1: 3$. The two microstructures with equiaxial and extended grains designed on a $200 \times 75 \times 200$ mesh with a step of $10 \mu \mathrm{m}$ are presented in Fig. 1. Polycrystal in Fig. 1(a) contains 1600 equiaxial grains with an average diameter of $120 \mu \mathrm{m}$. Microstructure in Fig. 1(b) consists of 450 grains with the average sizes across and along grain extension being of 120 and $360 \mu \mathrm{m}$, respectively.

To complete material definition, a constitutive model is assigned to each individual grain. The resolved shear stresses for aluminum alloys characterized by twelve slip systems slightly depend on the crystallographic orientation of grains. Therefore, we omit an explicit formulation of crystal plasticity but describe the elastic-plastic behavior of grains by a set of stress-strain curves scattered around an average curve. The elastic-plastic characteristics of equiaxial grains were varied within $10 \%$ about average values. Taking into account that cold rolling gives rise to the material texture characterized by the presence of grains with similar crystallographic orientations, the mechanical properties of extended grains were varied within 5\%. In addition, the elastic-plastic response of extended grains is assumed to be slightly different when load is applied along rolling and in a transverse direction (TD).

The elastic-to-plastic transition was defined by the von Mises criterion with accounting for the strain-hardening through the dependences fitting the experimental data for an A16061-T3 alloy [6]. 
In all calculations the bottom surface was a symmetry plane, its displacements were fixed in vertical direction and free in-plane. The top surface was free of external forces during loading. For both the equiaxial and extended grain aggregates the tensile load was applied to the front and back sides of the specimen along the $X_{2}$-axis (see Fig. 1). In the case of extended grains, an additional calculation for tension along $X_{1}$ direction was performed to compare deformation patterns realized under loading along the rolling direction and in the transverse direction. Lateral sides of the microvolumes were assigned with periodical boundary conditions which simulate constrained deformation inside the material volume.

\section{CALCULATION RESULTS}

Calculated surface images and corresponding surface profiles obtained for equiaxial and extended grain structures are presented in Fig. 2. Calculations show that surface roughness is strongly affected by the material texture. The surface patterns formed in equiaxial grain structure (Fig. 2(a)) and in a texture loaded across the grain extension (Fig. 2(c)) are very pronounced in comparison with the textured material loaded along rolling direction (Fig. 2(b)), the surface relief in the latter case being rather smooth. The surface folds formed at an early stage of plasticity do not change qualitatively, while their peak-to-valley distances progressively increase.

Let us analyse surface roughening in terms of micromechanics. From the viewpoint of classical macroscopic mechanics, in conditions of uniaxial tension there are no external forces acting across the tensile direction to cause out-of-plane displacements on the specimen free surface. It is, therefore, the internal microscopic stress-strain state alone which is responsible for the formation of the surface relief. Normal stress distributions in a subsurface section and at a depth of a half grain diameter below the free surface are shown in Fig. 3 for the equiaxial grain structure. The non-zero stresses are very low near the free surface and take place mainly along the grain boundaries (Fig. 3(a)). At a depth of a half grain diameter, however, they cover a whole section, demonstrating a quasi-periodic distribution of positive and negative values (Fig. 3(b)). Periodically distributed regions exposed to tensile and compressive stresses compensate each other and provide the total zero value of the averaged stress as it is dictated by the macroscopic equilibrium conditions. Acting from the inside the positive and negative stresses give rise to the formation of periodical ridges and valleys on the free surface.

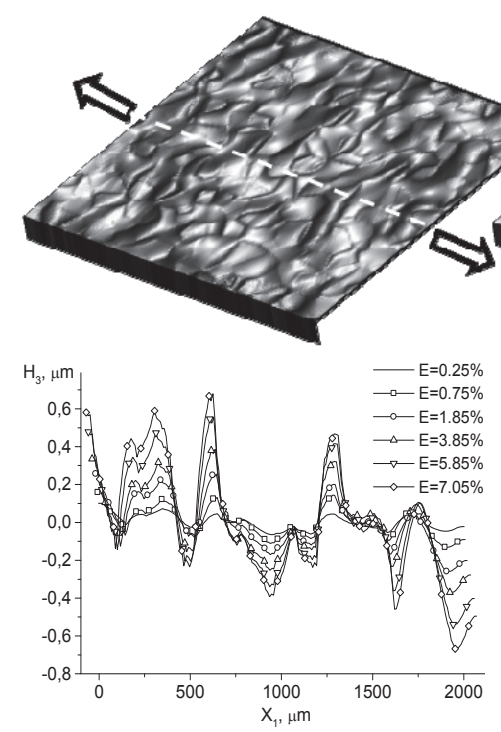

(a)

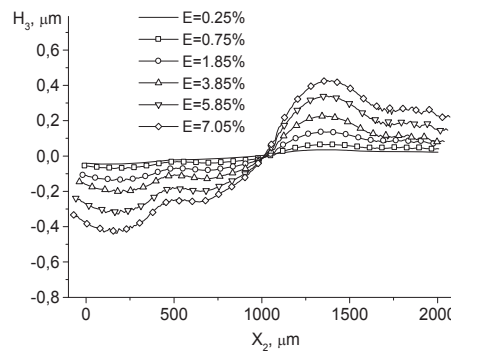

(b)

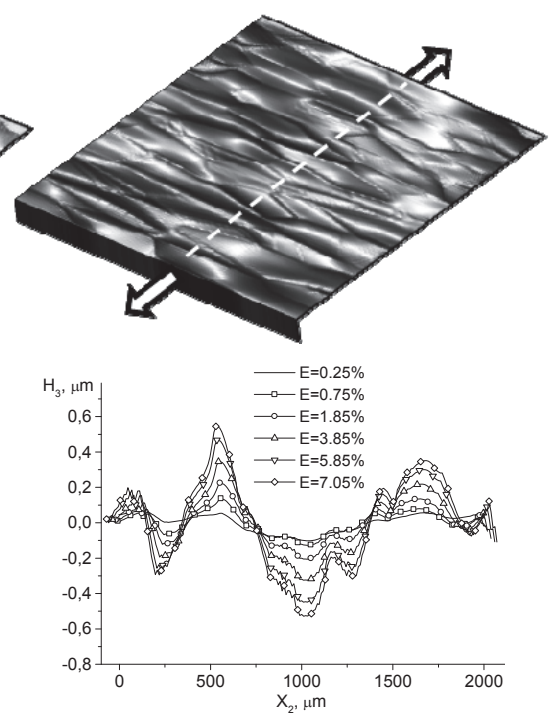

(c)

FIGURE 2. Calculated surface images and corresponding surface profiles along dotted lines in polycrystals with equiaxial (a) and extended grains loaded along (b) and across rolling direction (c) 




FIGURE 3. Stresses acting across the free surface along specimen centerlines at a depth of $5 \mu \mathrm{m}$ (a) and $50 \mu \mathrm{m}$ (b). Solid and dotted curves correspond to tension across and along rolling direction, respectively. Strain is $6 \%$

Analysis of the stress tensor components in polycrystals with extended grains has shown that when load is applied across the grain extension, both the normal stress and the shear stress associated with the plane of tension demonstrate higher values in contrast to the case when load is applied along rolling direction. As a result, severe surface roughening develops under the former kind of loading. The mechanical reason of this phenomenon is related to the grain shape effect. When load is applied across the grain extension, there are many grain boundaries crossed by the vector of external force. On the grain boundaries the force vector is decomposed into non-zero parts associated with the normal and shear stresses directed across the free surface. They give rise to the free surface roughening and related phenomena of plastic strain localization.

Although the stresses directed perpendicular to the free surface are rather low and so are the surface irregularities formed, even such a small roughness may critically affect the material properties and limit their usage. The situation may become even worse under cyclic loading where roughness gradually evolve giving rise to the plastic strain accumulation and localization.

\section{ACKNOWLEDGMENTS}

This work is supported by the Russian Foundation for Basic Research (14-08-00277-A) and a program to increase the competence of Tomsk Polytechnic University.

\section{REFERENCES}

1. O. Wouters, W. P. Vellinga, R. Van Tijum et al., Acta Mater. 53, 4043 (2005).

2. P. D. Wu and D. J. Lloyd, Acta Mater. 52, 1785 (2004).

3. D. Raabe, M. Sachtleber, H. Weiland, G. Scheele, and Z. Zhao, Acta Mater. 51(6), 1539 (2003).

4. M. Wilkins, Computer Simulation of Dynamic Phenomena (Springer, Berlin, 1999).

5. V. Romanova, R. Balokhonov, P. Makarov, et al., Comput. Mater. Sci. 28, 518 (2003).

6. V. Romanova, R. Balokhonov, and S. Schmauder, Mater. Sci. Eng. A 564, 255 (2013). 